\section{Prospective isolation of radiation induced erythroid stress progenitors reveals unique transcriptomic and epigenetic signatures enabling increased erythroid output}

\author{
Sofie Singbrant, ${ }^{1}$ Alexander Mattebo, ${ }^{1}$ Mikael Sigvardsson, ${ }^{2}$ Tobias Strid ${ }^{2}$ \\ and Johan Flygare ${ }^{1}$
}

${ }^{1}$ Division of Molecular Medicine and Gene Therapy, Lund Stem Cell Center, Lund University and ${ }^{2}$ Division of Molecular Hematology, Lund Stem Cell Center, Lund University, Lund, Sweden

ABSTRACT

M assive expansion of erythroid progenitor cells is essential for surviving anemic stress. Research towards understanding this critical process, referred to as stress-erythropoiesis, has been hampered due to the lack of specific marker-combinations enabling analysis of the distinct stress-progenitor cells capable of providing radioprotection and enhanced red blood cell production. Here we present a method for the precise identification and in vivo validation of progenitor cells contributing to both steady-state and stress-erythropoiesis, enabling for the first time indepth molecular characterization of these cells. Differential expression of surface markers CD150, CD9 and Sca1 defines a hierarchy of splenic stressprogenitors during irradiation-induced stress recovery in mice, and provides high-purity isolation of the functional stress erythroid burst-forming-units (stress-BFU-E) with a 100-fold improved enrichment compared to the stateof-the-art. By transplanting purified stress-progenitors expressing the fluorescent protein Kusabira Orange, we determined their kinetics in vivo and demonstrated that $\mathrm{CD} 150^{+} \mathrm{CD}^{+}{ }^{+} \mathrm{Sca1}^{-}$stress-BFU-E provide a massive but transient radioprotective erythroid wave, followed by multi-lineage reconstitution from $\mathrm{CD} 150^{+} \mathrm{CD} 9^{+} \mathrm{Sca} 1^{+}$multi-potent stem/progenitor cells. Whole genome transcriptional analysis revealed that stress-BFU-E express gene signatures more associated with erythropoiesis and proliferation compared to steady-state BFU-E, and are bone morphogenetic protein 4-responsive. Evaluation of chromatin accessibility through ATAC sequencing reveals enhanced and differential accessibility to binding sites of the chromatinlooping transcription factor CTCF in stress-BFU-E compared to steady-state BFU-E. Our findings offer a molecular insight into the unique capacity of stress-BFU-E to rapidly form erythroid cells in response to anemia and constitute an important step towards identifying novel erythropoiesis stimulating agents.

\section{Introduction}

Steady-state erythropoiesis is regulated mainly by changes in erythropoietin (EPO) levels that fine-tune survival and proliferation of erythroid colony-formingunits (CFU-E) and downstream precursor cells. In contrast, acute anemia induces a broader physiological response referred to as stress-erythropoiesis, which involves stimulation also of earlier progenitors to further increase the out-put of erythrocytes. This process is less characterized and mainly occurs in the murine spleen ${ }^{1}$ after seeding of progenitors from the bone marrow (BM)., ${ }^{2,3}$ Stress-erythropoiesis is differentially regulated, including increased responsiveness to additional factors like hypoxia, corticosteroids and bone morphogenetic protein 4 (BMP4). ${ }^{1,47}$ Importantly, stress-erythroid progenitors have the capacity to generate larger numbers of red blood cells than steady-state progenitors, and precise identification and enhanced understanding of their regulation are important steps towards discovering potential new erythroid-enhancing drugs for anemia treatment.

Ferrata Storti Foundation

Haematologica 2020

Volume 105(11):2561-2571

\section{Correspondence:}

JOHAN FLYGARE

johan.flygare@med.lu.se

SOFIE SINGBRANT

sofie.singbrant@med.lu.se

Received: August 14, 2019.

Accepted: January 2, 2020.

Pre-published: January 9, 2020.

doi:10.3324/haematol.2019.234542

(C)2020 Ferrata Storti Foundation

Material published in Haematologica is covered by copyright. All rights are reserved to the Ferrata Storti Foundation. Use of published material is allowed under the following terms and conditions:

https://creativecommons.org/licenses/by-nc/4.0/legalcode. Copies of published material are allowed for personal or internal use. Sharing published material for non-commercial purposes is subject to the following conditions: https://creativecommons. org/licenses/by-nc/4.0/legalcode, sect. 3. Reproducing and sharing published material for commercial purposes is not allowed without permission in writing from the publisher. 
While fluorescence-activated cell sorting (FACS)-based methods for fractionation of distinct erythroid progenitor cells in murine and human during steady-state ${ }^{8-11}$ has enabled in-depth characterization of mechanisms regulating steady-state erythropoiesis, ${ }^{11-15}$ the cells and mechanisms regulating stress-erythropoiesis remain poorly defined. To enable studies of stress-erythropoiesis we set out to identify novel marker-combinations separating and enriching for the early stress-progenitors mediating radioprotection and recovery from severe anemia. We previously demonstrated that fetal erythroid burst-formingunits (BFU-E) can be isolated as lineage$\mathrm{cKit}^{+} \mathrm{CD} 71 / \mathrm{CD} 24 \mathrm{a}^{\text {low }} \mathrm{Sca} 1^{-} \mathrm{CD} 34^{-}$with high purity from murine fetal liver, where erythropoiesis in many ways resemble stress-erythropoiesis. ${ }^{16}$ Attempts by other groups to isolate adult stress-erythroid progenitors from spleens of anemic mice and in vitro cultures have shown stress-BFU-E to be lineage-cKit ${ }^{+} \mathrm{CD} 71 / \mathrm{Ter} 119^{\text {low }}$, and further enriched in the $\mathrm{Sca} 1^{+} \mathrm{CD} 34^{-} \mathrm{CD} 133^{-}$fraction. However, very few of these cells possess BFU-E potential $(0.1-0.2 \%)$. Furthermore, in the active debate on lineage potential of stem- and progenitor cells, genuine megakaryocytic/erythroid potential is often overlooked since mature erythrocytes and platelets are difficult to trace in vivo after transplantation. Hence, the identity of pure stress-BFU-E remains largely elusive.

Using a novel combination of surface markers together with the tracing marker Kusabira Orange which is expressed in all cells, we have developed a method for high purity fractionation of a hierarchy of multi-potent progenitors, stress-BFU-E, and stress-CFU-E within the lineage-cKit ${ }^{+} \mathrm{CD} 71 / \mathrm{CD} 24 \mathrm{a}^{\text {low }}$ cells in spleen during irradiation-induced stress-erythropoiesis as well as in steadystate BM, and for the first time determined their kinetics and full differentiation potential in vivo. The formation of stress-BFU-E was highly dependent on functional BMPsignaling, and stress-BFU-E displayed enhanced expression of BMP-responsive genes, as well as gene signatures associated with erythropoiesis and proliferation compared to their steady-state counterpart. In addition, discrepancies in the epigenetic landscape were selectively enriched for putative binding sites for the chromatin-looping transcription factor CTCF. In conclusion, our findings provide high-purity isolation of both steady-state BFU-E and the stress-BFU-E mediating recovery from severe anemia, and offer molecular insight to and functional determination of the unique capacity of stress-BFU-E to rapidly form erythroid cells in response to anemia.

\section{Methods}

\section{Mice and transplantations}

All procedures involving mice were approved by the Animal Ethics Committee of Malmö/Lund, Sweden. Anemia was induced by lethally irradiating 8-12 week-old recipient mice (C57Bl/6; Ly5.2) with a split dose of $2 \times 500 \mathrm{cGy}$, followed by transplantation of $2 \times 10^{6}$ unfractionated BM cells to rescue and trigger stress-erythropoiesis. Donor mice (B6SJL; Ly5.1) were either Kusabira Orange $(\mathrm{KuO})$ positive or negative. Recipients were sacrificed on day 8 for analysis of stress recovery in the spleen.

For in vivo tracing, 500 multipotent progenitors (sMPP), 5,000 sBFU-E or 5,000 sCFU-E, all $\mathrm{KuO}^{+}$, were FACS-sorted from day 8 stressed spleens and transplanted into lethally irradiated secondary recipients together with 105 unfractionated wild-type
BM cells as support. Secondary recipients were bled at 1, 2 and 4 weeks, and sacrificed at 2 or 4 weeks post transplantation for analysis of lineage potential and kinetics in peripheral blood (PB), $\mathrm{BM}$ and spleen.

\section{Flow cytometry}

A complete description of all antibodies used is listed in the Online Supplementary Materials and Methods.

\section{Hematopoietic progenitor assays}

All colony assays were incubated at $37^{\circ} \mathrm{C}$ incubators in $5 \%$ $\mathrm{CO} 2$ with either $21 \%$ or $1-4 \% \mathrm{O}_{2}$ as indicated, and scored on day 4 (CFU-E) or 7-8 (BFU-E and mixed colonies).

\section{RNA sequencing}

Splenic stress- and BM steady-state progenitors were FACSsorted in triplicates. Strand specific RNA-sequencing libraries were constructed using SMARTer Stranded Total RNA-Seq Kit v2 (Takara Bio) followed by sequencing on a HiSeq3000 (Illumina).

\section{Assay for Transposase Accessible Chromatin sequencing}

3,000 splenic stress- and BM steady-state BFU-E were FACSsorted in triplicates for the assay of Transposase Accessible Chromatin (ATAC) library preparation and sequencing as described previously. ${ }^{18}$ Libraries were subject to single-end sequencing on a NextSeq500 (Illumina).

\section{Statistical analysis}

For all statistical analysis, apart from RNA- and ATAC-sequencing for which the specifications are stated in the Online Supplemental Materials and Methods, statistical significance was calculated using ANOVA accounting for multiple comparisons, followed by Tukey's multiple comparisons test. One-way ANOVA was used for single time point analysis and Two-way ANOVA was used when measuring potential over time (Figure 2). ${ }^{*} P \leq 0.05,{ }^{* *} P \leq 0.01,{ }^{* * *} P \leq 0.001,{ }^{* * *} P \leq 0.0001$.

A detailed description of all methods used is available online in the Online Supplementary Materials and Methods.

\section{Results}

CD150, CD9 and Sca1 identify a hierarchy of splenic stress-progenitors during irradiation-induced stress recovery

To identify the stress-progenitors involved in irradiation-induced anemia and stress recovery, we subjected mice to lethal irradiation followed by BM transplantation and analyzed recipient spleens on day 8 (Figure 1A) when the greatest expansion of stress-progenitors occurs. ${ }^{3}$ Using this model, stress-progenitors were previously identified as $\mathrm{Lin}^{-} \mathrm{CKit}^{+} \mathrm{CD} 71 / \mathrm{Ter} 19^{\text {low }}$, although at low frequencies $(0.2 \%){ }^{3}$ To further enrich for stress-erythroid progenitors we included CD150, known to mark megakaryocytic/erythroid progenitors during steady-state hematopoiesis. ${ }^{9}$ All BFU-E potential in the $\mathrm{cKit}{ }^{+} \mathrm{CD} 71^{\text {low }} / \mathrm{Ter} 119^{\text {low }}$ population resided in the $\mathrm{CD}_{150^{+}}$fraction (Figure 1B-C). Extramedullary expansion of early erythroid progenitors during stress-erythropoiesis is reminiscent of fetal liver erythropoiesis. We therefore analyzed previously published mRNA expression data (GSE26086) ${ }^{16}$ from E14.5 to E15.5 fetal liver erythroid progenitors to identify possible additional markers for further sub-division of adult stresserythroid progenitors (Online Supplementary Figure S1A). 
A

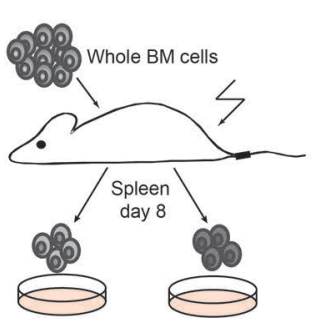

B

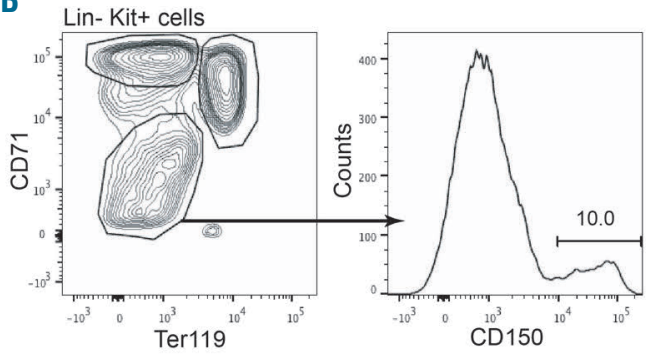

C

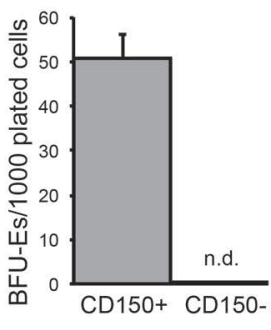

D

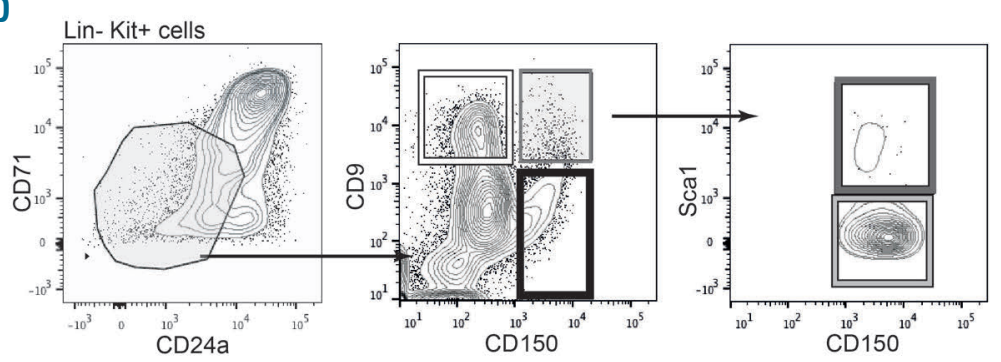

口 CD150-CD9+

口 CD150+CD9+Sca1-

C CD150+CD9+Sca1+

CD150+CD9-

E
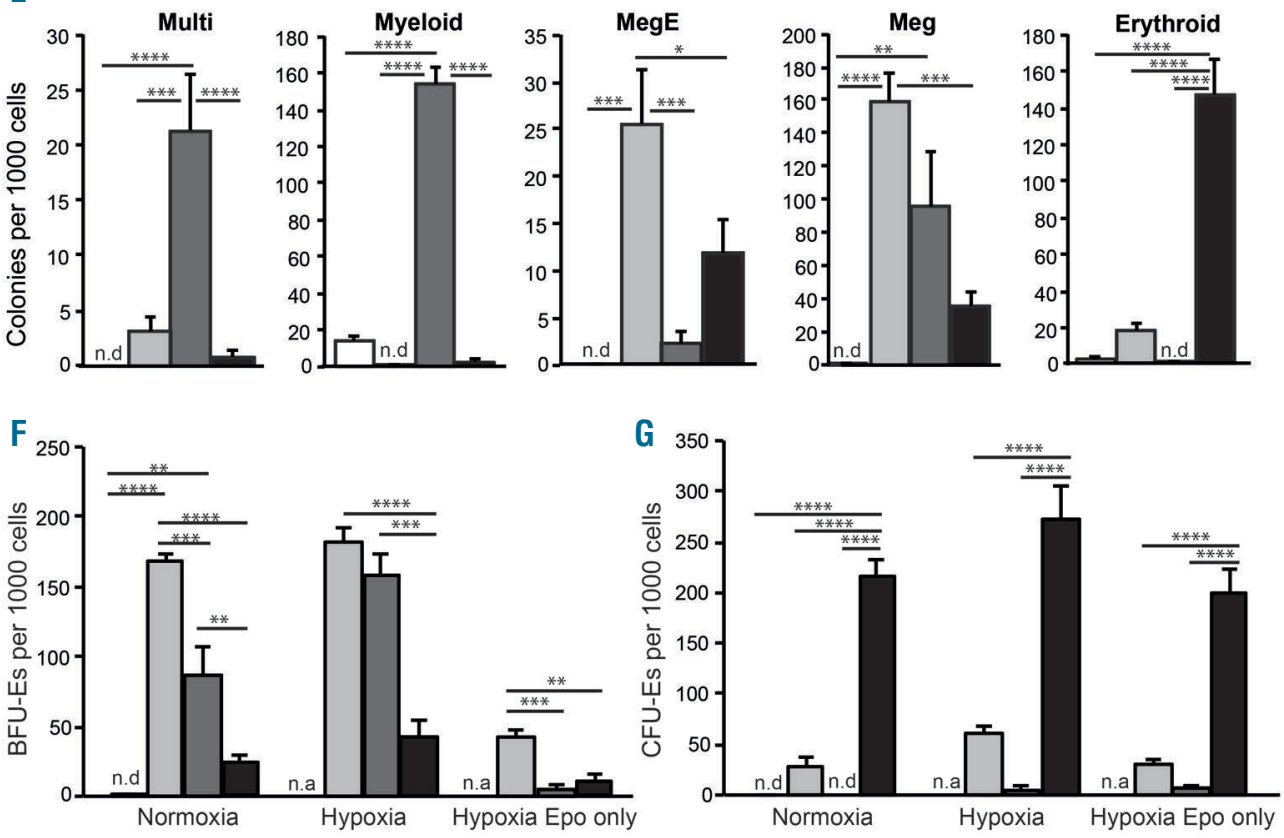

H
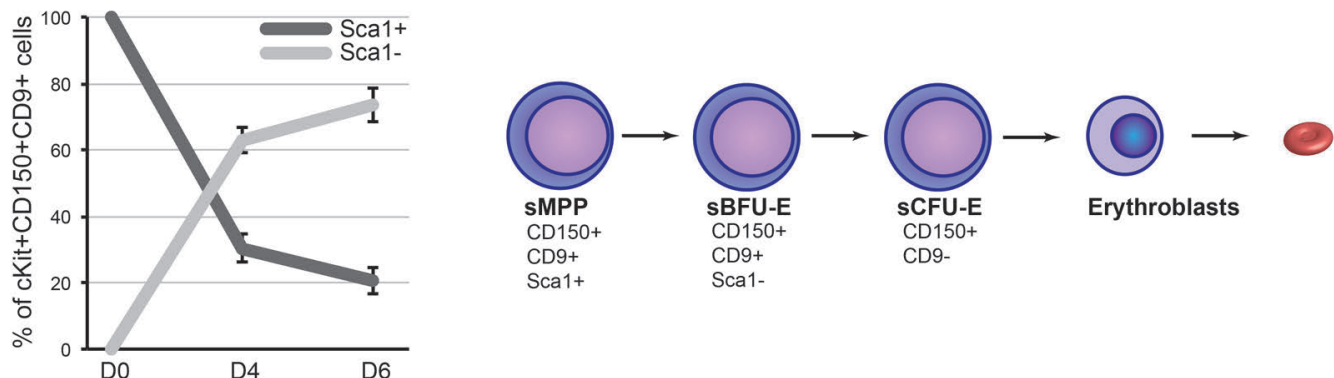

Figure 1. CD150, CD9 and Sca1 identify a hierarchy of splenic stress-progenitors during irradiation-induced stress recovery. (A) Stress-erythropoiesis was induced using lethal irradiation followed by transplantation of unfractionated bone marrow (BM), and splenic stress-progenitors were assessed on day 8 using colony assays. (B) Gating strategy and (C) BFU-E potential of FACS-sorted stress-progenitors from spleen day 8 based on CD150 expression ( $n=5$ ). (D) Gating strategy for further fractionation of stress-progenitors within lineage-Kit' cells using CD150 and CD9 and Sca1. (D-G) Colony forming capacity of splenic day 8 stress-progenitors, FACSsorted based on (D) CD150/CD9 (normoxia, $n=4$ ), and (E-G) CD150/CD9/Sca1 ( $=7$ for normoxia, $n=4$ for hypoxia) within the Lin-cKit ${ }^{+} C D 71^{\text {low }} C D 24^{\text {low }}$ fraction. The cells were incubated in normoxia (21\% 02) or hypoxia (1\% 02) as indicated and scored on day 4 (CFU-E) or day 78 (mixed and BFU-E) - (H) FACS sorted cells $150 *$ ( CD150 of novel sur $\mathrm{E}$ (SCFU-ES), defining a stress-progenitor hierarchy in spleen during irradiation-induced stress recovery, where Sca1 and subsequently CD9 are downregulated with increased differentiation. Data displayed as average \pm standard error of the mean (SEM), n.d: not detectable, n.a: not applicable, $* P \leq 0.05, * * P \leq 0.01, * * * P \leq 0.001$,
$\star * * * P \leq 0.0001$. 
As previously reported by us in fetal liver, ${ }^{16}$ exclusion of mature erythroid precursor cells in spleen was facilitated by inclusion of CD24a as a negative selection marker (Online Supplementary Figure S1B). Of the surface markers tested (CD9, CD11a, CD34, CD48, CD63, and CD79b), only CD9 could further fractionate the BFU-E-containing $\mathrm{CD} 150^{+}$population in stressed spleen (Online Supplementary Figure S1C). CD133 was used in a recent study together with CD34 to fractionate stress-progenitor potential of cultured $\mathrm{Sca} 1^{+} \mathrm{CKit}{ }^{+} \mathrm{CD} 71 / \mathrm{Ter} 1191^{\text {ow }}$ cells. ${ }^{17}$ However, neither CD34 nor CD133 could further fractionate $\mathrm{CD} 150^{+}$stress-progenitors (Online Supplementary Figure S1C and Online Supplementary Figure S2A, respectively). To further discriminate putative multi-potent stress-progenitors from lineage restricted stress-BFU-E we also included Sca1, which in steady-state BM separates Sca1 ${ }^{+}$ hematopoietic stem cells (HSC) and Sca1- myelo-erythroid progenitors. ${ }^{9}$ Analysis of $\mathrm{Lin}^{-} \mathrm{cKit}^{+} \mathrm{CD} 71^{\text {low }} / \mathrm{CD} 24 \mathrm{a}^{\text {low }}$ splenic stress-progenitors fractionated based on
CD150/CD9/Sca1 expression (Figure 1D), clearly demonstrated that the majority of multi-lineage, megakaryocytic and BFU-E colony forming potential resided in the $\mathrm{CD} 150^{+} \mathrm{CD}^{+}$population (Figure $1 \mathrm{E}-\mathrm{F}$ ). Stress-progenitors negative for CD9 gave rise to more mature erythroid colonies (Figure 1E, G), which was also true for CD133 cells (Online Supplementary Figure S2B), whereas CD150cells mainly gave rise to myeloid colonies (Figure 1E). Only Sca1 expressing $\mathrm{CD}_{150}{ }^{+} \mathrm{CD}^{+} \quad \mathrm{cKit}^{+}$ CD71 $1^{\text {low }} / \mathrm{CD} 24 a^{\text {low }}$ stress-progenitors (about $10 \%$ ) gave rise to mixed and myeloid colonies (Figure 1E), while megakaryocytic/erythroid potential was retained in $\mathrm{Sca}^{-}$stress-progenitors (Figure 1E-F). Stress-BFU-E are reported to form BFU-E colonies with Epo alone, ${ }^{1}$ but require hypoxia and additional cytokines for maximum expansion. ${ }^{3}$ Colony assays demonstrated that BFU-Eforming potential resided almost exclusively in the $\mathrm{CD} 150^{+} \mathrm{CD}^{+}$population (Figure $1 \mathrm{~F}$ ). Importantly, as many as $21.4 \pm 2.2 \%$ of $\mathrm{CD} 150^{+} \mathrm{CD}^{+}$cells gave rise to $\mathrm{BFU}-\mathrm{E}$
A

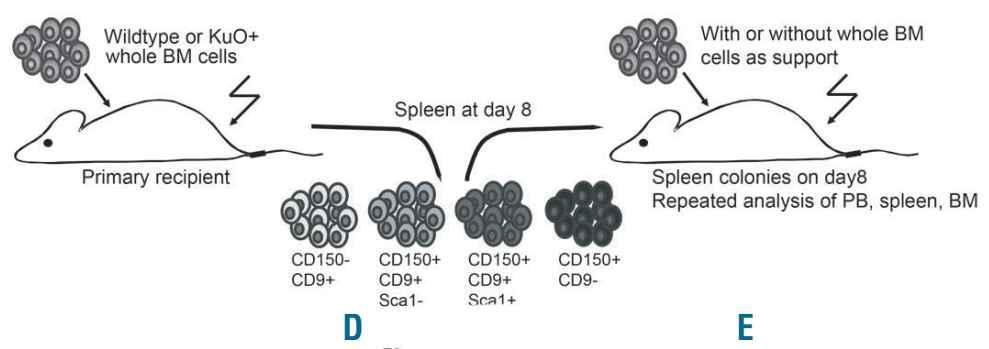

C

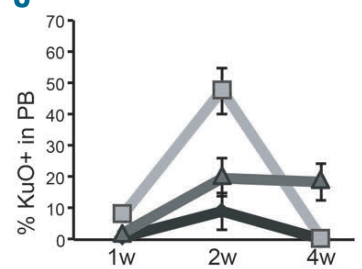

G

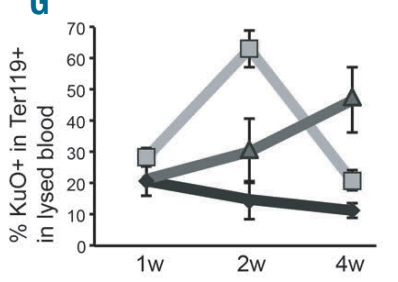

D

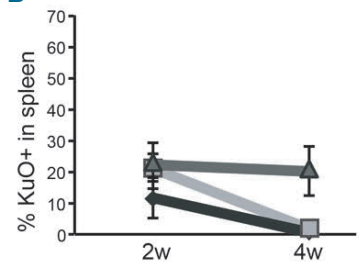

$\mathrm{H}$

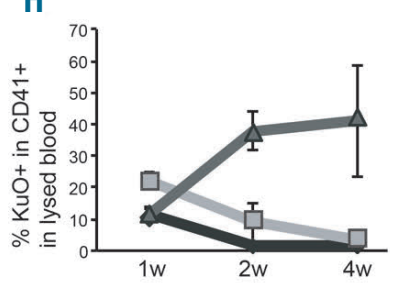

$\mathrm{E}$
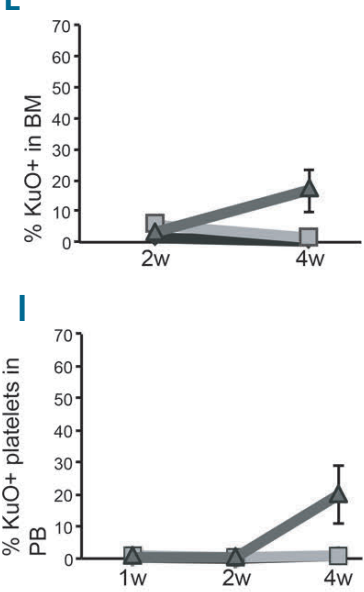

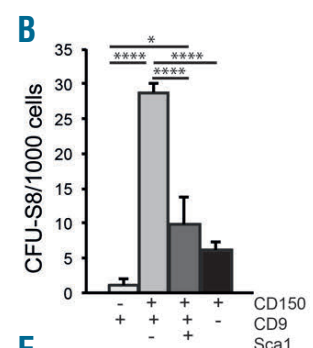

F
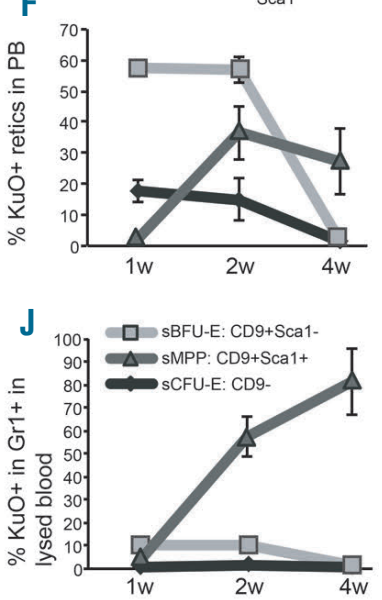

K

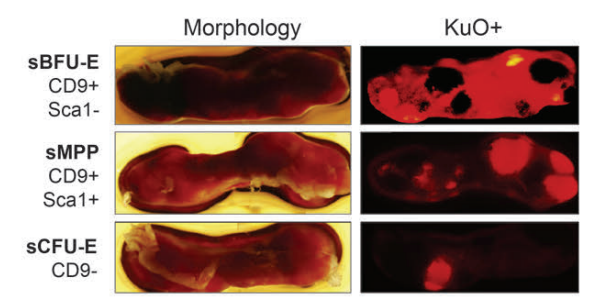

Figure 2. Stress-BFU-E provide a transient wave of primarily erythroid cells, followed by multi-lineage reconstitution from stress-MPP. (A) Stress-erythropoiesis was induced using lethal irradiation followed by transplantation of unfractionated bone marrow (BM) from (B) wild-type or (C-K) transgenic Kusabira Orange (KuO) mice. Splenic stress-progenitor populations were FACS-sorted on day 8, transplanted into lethally irradiated secondary recipients (B) without support to evaluate spleen colony formation (CFU-S8) ( $n=3-7 ; 600$ cells per recipient without support) or (C-K) together with 105 unfractionated wild-type BM support cells to monitor their repopulation capacity in vivo over time in (C) peripheral blood (PB), (D) spleen and (E) BM, as determined by KuO fluorescence and FACS ( $n=6$ at $1-2$ weeks and $n=3$ at 4 weeks; 500 sMPP or 5,000 of each stress-erythroid progenitor per recipient, all with 105 wild-type support BM cells). (F-J) Contribution of sorted Ku $0+$ progenitors in PB to (F) reticulocytes (whole PB), (G) Ter119 ${ }^{+}$erythroid cells, (H) CD41+ cells, (I) platelets (whole PB), and (J) Gr1+ myeloid cells in PB, as determined by FACS (after lysis of red blood cells if not stated otherwise). (K) Representative picture of whole spleens 2 weeks after transplantation assessing KuO contribution using epifluorescence microscopy and green filtered laser excitation. Data displayed as average \pm standard error of the mean $(\mathrm{SEM}), * P \leq 0.05, * * P \leq 0.01, * * * P \leq 0.001$, $* * * * P \leq 0.0001$. 
colonies, providing over 100 -fold improved purity compared to the state-of-the-art (0.1-0.2\%). Although both $\mathrm{Sca}^{-}$and $\mathrm{Sca}^{+}$cells formed BFU-E colonies when stem cell factor (SCF) was present, only Sca1- cells formed BFU$\mathrm{E}$ in erythropoietin (Epo) alone (Figure 1F). Furthermore, $\mathrm{Sca}^{-}$progenitors generally gave rise to larger colonies than $\mathrm{Sca}^{+}$progenitors (data not shown). A notable $27.4 \pm 3.2 \%$ of $\mathrm{CD} 150^{+} \mathrm{CD}^{-}$cells gave rise to CFU-E, which were mainly independent of hypoxia and SCF (Figure 1G). Although the number of colonies was not affected, hypoxia resulted in increased proliferation and larger colonies (data not shown). FACS-sorted
A

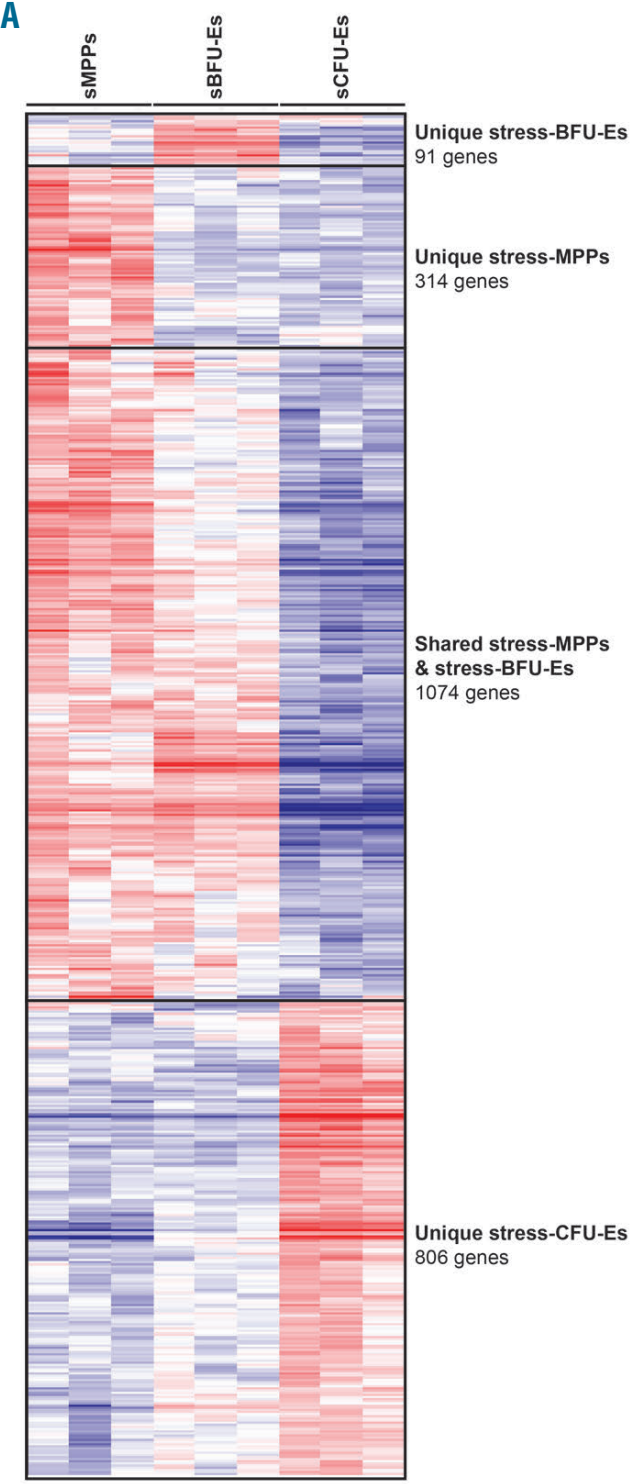

C

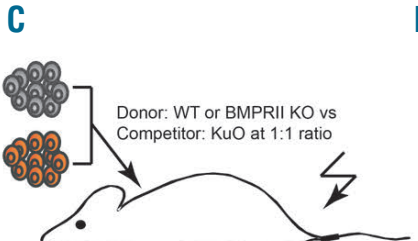

D

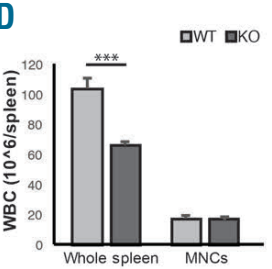

B

Cluster specific cellular processes
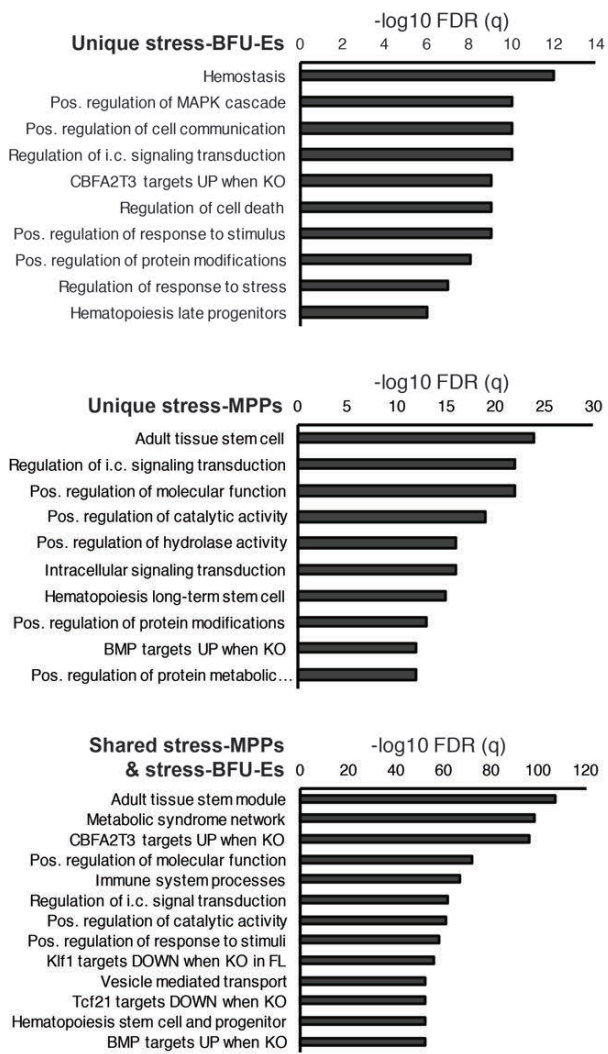

$-\log 10$ FDR $(q)$
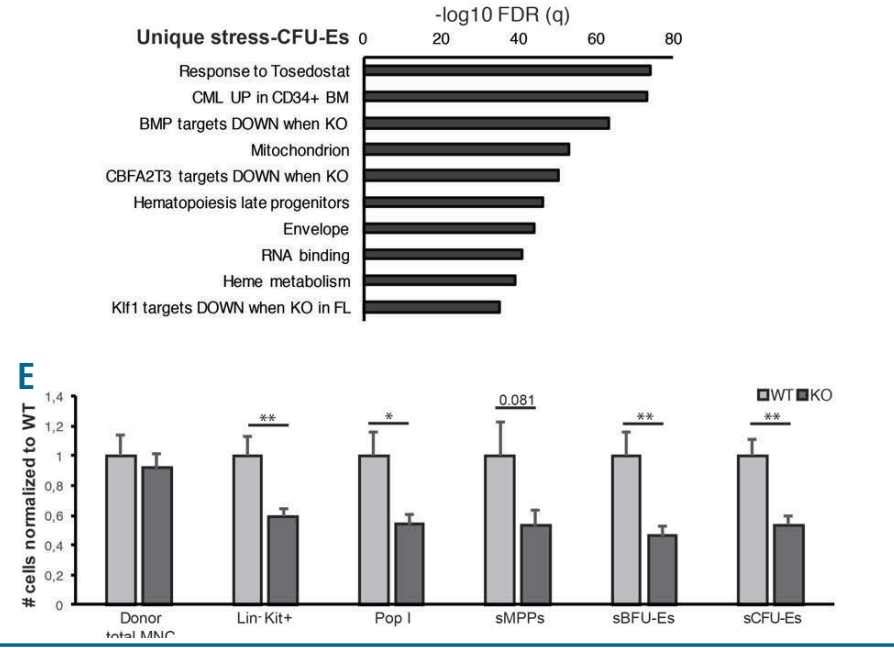

Figure 3. Stress-BFU-E and stress-CFU-E display opposing expression patterns of BMP- and CBFA2T3-responsive genes. (A) Unsupervised clustering of RPKM normalized data on genes with a significantly different expression between any of the samples (FDR $<0.05$, $\log _{2}$ fold change $>0.58$ ) from RNA-sequencing of FACS sorted stress-progenitor populations as indicated ( $n=3$, for further details see the Methods section). (B) Cellular processes for each of the clusters generated in (A), as analyzed by GSEA. For a complete gene lists for each cluster see the Online Supplementary Table S1. (C) Experimental outline of competitive transplantation of wild-type or BMP receptor II (BMPRII) deficient bone marrow against $\mathrm{KuO}^{+}$wild-type bone marrow. The stress recovery was analyzed in spleens of recipients on day 8 after transplantation ( $n=7$ per group). (D) White blood cell count (WBC) per spleen before and after enrichment of mononuclear cells (MNC). (E) Detailed analysis of donorderived (KuO-) MNC and stress-populations within MNC spleen cells as indicated. Data displayed as average \pm standard error of the mean (SEM) of total number of cells/spleen (D) and normalized to wild-type (E), ${ }^{*} P \leq 0.05, * * P \leq 0.01$. 
$\mathrm{CD}_{150} \mathrm{CD}^{+} \mathrm{Sca}^{+}$progenitors gave rise to $\mathrm{CD} 150^{+} \mathrm{CD}^{+} \mathrm{Sca}^{-}$in culture (Figure $1 \mathrm{H}$ ), indicating that more restricted Sca1- progenitors arise from multi-potent Sca1+ progenitors. Taken together, differential expression of cell surface markers CD150, CD9 and Sca1 defines a hierarchy of splenic $\mathrm{Lin}^{-} \mathrm{CKit}{ }^{+} \mathrm{CD} 71 / \mathrm{CD} 24 \mathrm{a}^{\text {low }}$ stress-progenitors during irradiation-induced stress recovery in mice (Figure $1 \mathrm{I}$ ). The stress-BFU-E, forming BFU-E colonies in vitro $\left(\mathrm{CD} 150^{+} \mathrm{CD}^{+} \mathrm{Sca} 1^{-}\right.$, hereafter referred to as stress-BFU-E or sBFU-E), can be separated from multi-potent stress-progenitors $\left(\mathrm{CD} 150^{+} \mathrm{CD} 9^{+} \mathrm{Sca} 1^{+}\right.$, hereafter referred to as stress-MPP or sMPP) and stressCFU-E (CD150+CD9-, hereafter referred to as stress-CFUE or sCFU-E) by Sca1 and CD9 expression respectively.

\section{Stress-BFU-E provide a transient wave of primarily} erythroid cells, followed by multi-lineage reconstitution from stress-MPP

To determine the kinetics and full in vivo potential of the identified stress-progenitors, we used a transgenic mouse that constitutively expresses the fluorescent protein Kusabira Orange $(\mathrm{KuO})$ in all cells, including erythrocytes and platelets. ${ }^{20}$ Lethally irradiated recipients were transplanted with $\mathrm{BM}$ from $\mathrm{KuO}$ mice, and $\mathrm{KuO}^{+}$splenic stressprogenitors isolated on day 8 were subsequently transplanted into secondary recipients, either without support cells to score spleen colony-forming-units day 8 (CFUS8), ${ }^{21}$ or together with $10^{5}$ unfractionated wild-type BM support cells to monitor their in vivo repopulation capacity over time (Figure 2A). sBFU-E demonstrated the highest CFU-S8 potential (Figure 2B), indicative of robust shortterm radio-protective capacity. Analysis of overall repopulation potential of sorted $\mathrm{KuO}^{+}$progenitors demonstrated
Table 1. Top 20 up-regulated genes in stress-BFU-E compared to steady-state.

\begin{tabular}{lcc} 
Gene symbol & log2 fold change & -10 10 FDR \\
Ctse & 2,254 & 19,109 \\
Myh10 & 1,542 & 7,858 \\
\hline Aldh1a1 & 1,529 & 5,762 \\
Pop1 & 1,178 & 2,814 \\
\hline Tfrc & 1,171 & 4,072 \\
Psat1 & 1,133 & 3,136 \\
\hline Timm10b & 1,123 & 2,505 \\
Vkorc1 & 1,119 & 2,652 \\
\hline Sipa1 & 1,117 & 5,900 \\
Tmem70 & 1,082 & 2,401 \\
\hline Usp14 & 1,063 & 6,477 \\
Emilin2 & 1,062 & 3,012 \\
\hline Josd1 & 1,039 & 3,305 \\
Psmb2 & 1,036 & 2,079 \\
\hline Suclg1 & 1,035 & 5,608 \\
Trip13 & 1,014 & 2,078 \\
\hline Arap2 & 1,005 & 2,132 \\
Tmem201 & 1,001 & 2,320 \\
\hline Mob1b & 0,992 & 2,190 \\
Atl2 & 0,984 & 3,323
\end{tabular}

The most highly up-regulated genes in sBFU-E compared to steady-state BFU-E were Ctse/Cathepsin E: an erythrocyte membrane aspartic proteinase previously described as a down-stream target of Foxo3 in erythroid regulation ${ }^{38}$ Myh10: a non-muscle myosin involved in cell division of erythroblasts and other cells ${ }^{39}$ Aldh1a1: a critical enzyme involved in metabolism of reactive oxygen species and retinoic acid shown to mark adult definitive erythroid fate in mouse and human blood development, and the transferrin receptor Tfrc/CD71.
A

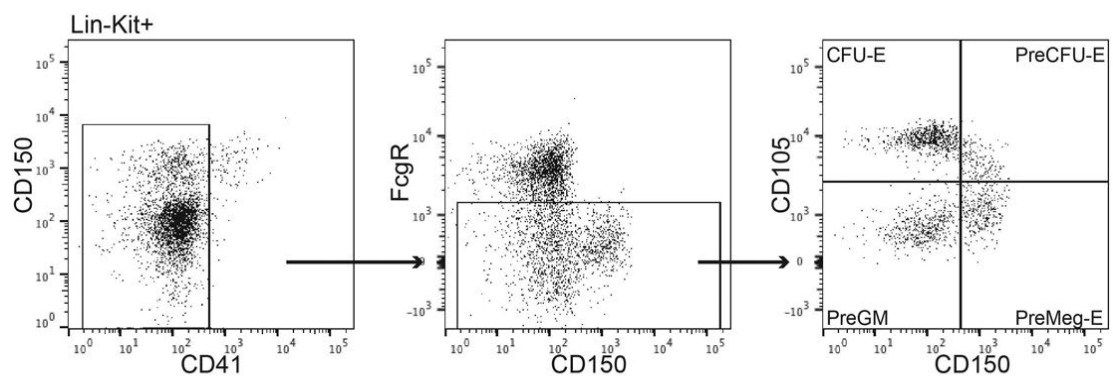

C

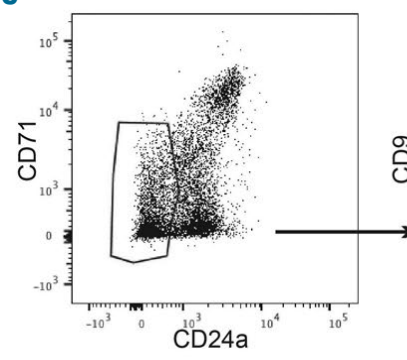

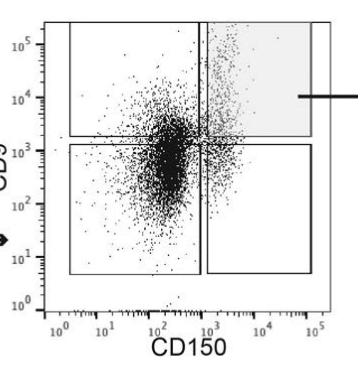

B

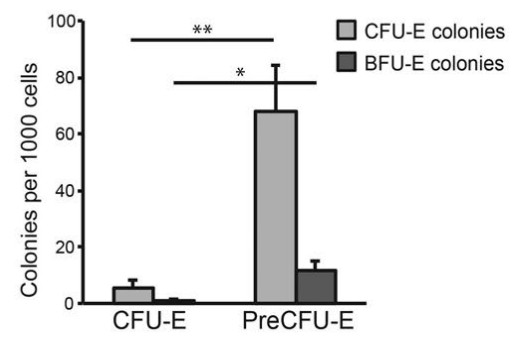

D

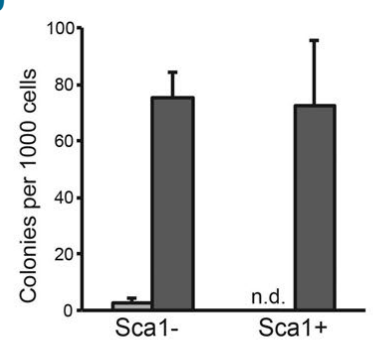

Figure 4. CD150 and CD9 mark BFU-E potential during steady-state erythropoiesis, providing improved identification of myelo-erythroid progenitors in the bone marrow. Gating strategy and colony forming potential (CFU-E; light grey, BFU-E; dark grey) of FACS sorted (A-D) steady-state bone marrow progenitors based on (A-B) classical fractionation using CD150 and CD1059, and (C-D) the new markers CD150/CD $/$ Sca1. All cells were incubated in $4 \% \mathrm{O}_{2}$, and Scored on day 4 (CFU-E) or day 7-8 (BFU-E). Data displayed as average \pm standard error of the mean (SEM), n.d: not detectable, $* P \leq 0.05, * * P \leq 0.01$. 
that sBFU-E and sCFU-E provide a transient wave of reconstitution in $\mathrm{PB}$ and spleen, with limited contribution to BM hematopoiesis. In contrast, sMPP increased their output over time, and was the only population that efficiently populated the $\mathrm{BM}$ and still contributed to hematopoiesis at four weeks (Figure 2C-E).

At two weeks $47.4 \pm 7.6 \%$ of $\mathrm{PB}$ cells were $\mathrm{KuO}^{+}$in mice transplanted with sBFU-E, compared to $9.0 \pm 5.9 \%$
$(P \leq 0.0001)$ for sCFU-E (Figure 2C). Analysis of the differentiation potential of transplanted stress-progenitors revealed that sBFU-E gave rise to $57.4 \pm 1.9 \%$ of reticulocytes compared to $17.5 \pm 3.5 \% \quad(P \leq 0.0001)$ for $\mathrm{sCFU}-\mathrm{E}$, while reticulocytes from sMPP were barely detected (Figure 2F; PB 1 week). Furthermore, the initial overall formation of reticulocytes was 2 -fold higher in mice transplanted with sBFU-E compared to sCFU-E (Online

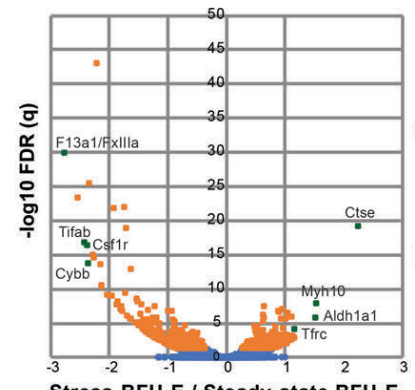

Stress-BFU-E / Steady-state BFU-E (log2 FoldChange)
B
C

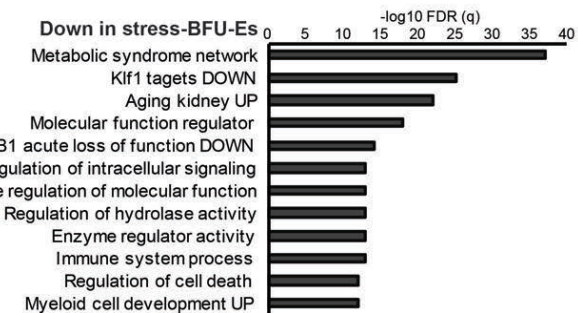

D

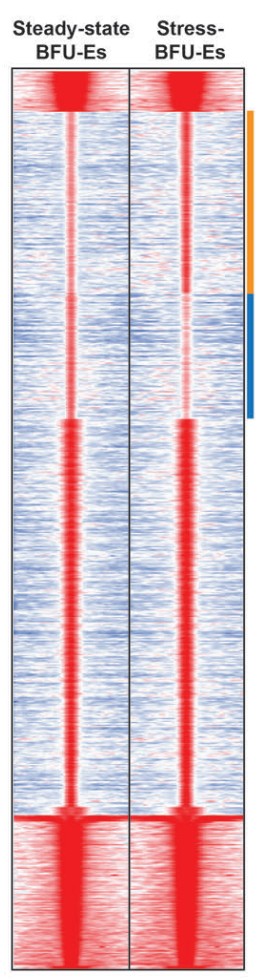

$\mathrm{E}$ Peaks enriched in stress-BFU-Es Rank Motif P-value \% of target \% of background 1 GCCCCCTAGTGG $1 \mathrm{e}-2376 \quad 22.85 \% \quad 2.73 \%$ 2 ACTTCCTETE $\quad 1 \mathrm{e}-913 \quad 31.56 \% \quad 12.88 \%$ 3 SAACCACAA $1 \mathrm{e}-530 \quad 24.67 \% \quad 11.40 \%$

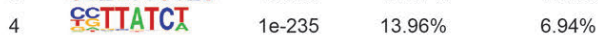

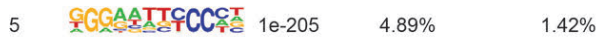

F Peaks enriched in steady-state BFU-Es

\begin{tabular}{|c|c|c|c|c|}
\hline Rank & Motif & P-value & $\%$ of target & $\%$ of background \\
\hline 1 & ACTTCCTCTI & $1 e-500$ & $28.98 \%$ & $12.73 \%$ \\
\hline 2 & TGTGGTITT웅 & $1 \mathrm{e}-237$ & $28.73 \%$ & $16.83 \%$ \\
\hline 3 & GCCCCCTAGTGG & $1 e-178$ & $2.97 \%$ & $0.42 \%$ \\
\hline 4 & TGCTGAGTCATE & $1 e-74$ & $2.70 \%$ & $0.82 \%$ \\
\hline & 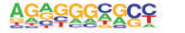 & $1 \mathrm{e}-72$ & $5.40 \%$ & $2.49 \%$ \\
\hline
\end{tabular}

G

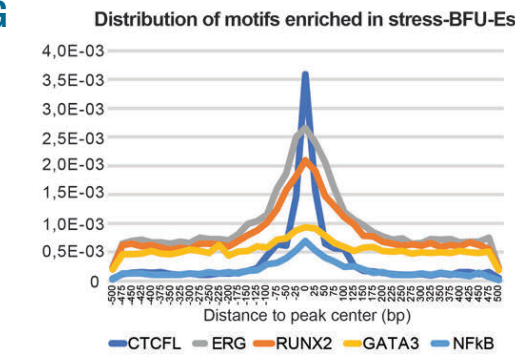

H

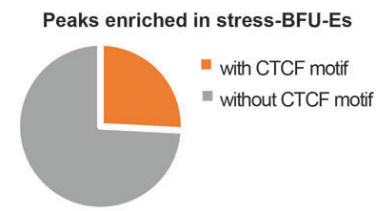

\section{Best match/Details}

BORIS(Zf)/K562-CTCFL-ChIP-Seq(GSE32465)/Homer(0.934) ERG(ETS)/NCaP-ERG-ChIP-Seq(GSE14097)/Homer(0.956) RUNX2(Runt)/PCa-RUNX2-ChIP-Seq(GSE33889)/Homer(0.971) GATA3(Zf)/iTreg-Gata3-ChIP-Seq(GSE20898)/Homer(0.981) NFkB-p65(RHD)/GM12787-p65-ChIP-Seq(GSE19485)/Homer(0.925)

\section{Best match/Details}

PB0058.1_Sfpi1_1/Jaspar(0.958)

RUNX2(Runt)/PCa-RUNX2-ChIP-Seq(GSE33889)/Homer(0.975) BORIS(Zf)/K562-CTCFL-ChIP-Seq(GSE32465)/Homer(0.925) NF-E2(bZIP)/K562-NFE2-ChIP-Seq(GSE31477)/Homer(0.977) BORIS(Zf)/K562-CTCFL-ChIP-Seq(GSE32465)/Homer(0.725)

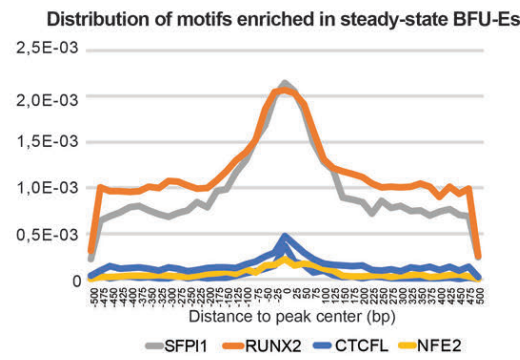

Peaks enriched in steady-state BFU-Es

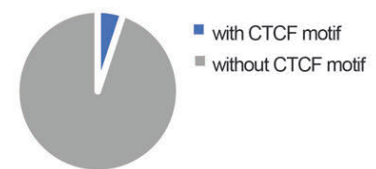

Figure 5. Compared to steady-state BFU-E, stress-BFU-E have enhanced expression of genes associated with BMP signaling, erythropoiesis and proliferation, and have enhanced and differential accessibility to CTCF binding sites. (A) RNA-sequencing was conducted on BFU-E FACS-sorted from steady-state bone marrow and day 8 stress spleens respectively $(n=3)$. Significant differential expression was determined as $-\log _{10}$ FDR $>2$ and $\log _{2}$ fold change $>0.58$, for a full list of differentially expressed genes see the Online Supplementary Table S2. (B-C) Cellular processes for genes that were (B) up- and (C) down-regulated in stress-BFU-E compared to steady-state respectively, as analyzed by GSEA. (D) Fishbone heatmap outlining specific and shared ATAC-seq peaks in steady-state and stress BFU-E respectively. Each row represents one peak, and the color represents the intensity of chromatin accessibility. Peak files for heatmap were created in HOMER44 and grouped based on K-means clustering (3-clusters, 100-runs) performed in Cluster3, revealing clusters of peaks enriched in stress- (orange) and steady-state BFU-E (blue) respectively. (E-F) Motif analysis was performed on stress (E) and steady-state (F) enriched regions from peak lists extracted from panel D using findMotifsGenome.pl in HOMER. (G) Distribution of identified top motifs was investigated +/- 500 bp from corresponding peak centers. (H) Annotation of the CTCF motif within stress(orange) and steady-state (blue) BFU-E enriched peaks respectively. 
Supplementary Figure 3A), albeit from low levels, with virtually no contribution to reticulocytes from sMPP during the first week (Online Supplementary Figure 3B). sBFU-E also dominated the overall contribution to erythroid cells during the first two weeks of recovery (Figure 2G, $P \leq 0.0001$ and $P \leq 0.01$, respectively.). All cell populations gave rise to $\mathrm{CD} 41^{+}$cells short after transplantation (Figure $2 \mathrm{H}$ ), whereas only sMPP produced platelets, which displayed slower kinetics compared to the formation of erythroid cells (Figure 2I; $P \leq 0.0001$ ). In agreement with the colony formation ability in vitro, myeloid (Figure 2J) as well as lymphoid lineages (Online Supplemental Figure S3C-D) were mainly derived from sMPP.

The $\mathrm{KuO}^{+}$blood lineage distribution and repopulation kinetics in spleen after transplantation was largely reflected by that observed in PB (Online Supplemental Figure S4), while the BM had a considerably slower repopulation pattern (Online Supplemental Figure S5).

Morphological analysis of spleens at 2 weeks post transplantation revealed a strikingly different distribution of repopulating cells (Figure $2 \mathrm{~K}$ ). Progeny from sBFU-E was evenly distributed throughout the spleen with occasional $\mathrm{KuO}^{-}$clones possibly arising from the support BM. In contrast, sMPP gave rise to a limited number of large clones, consistent with spleen colonies formed by more primitive stem/progenitor cells (CFU-S12). ${ }^{23}$ Taken together, in vivo tracing demonstrated clear separation of functionally distinct populations within $\mathrm{CKit}{ }^{+} \mathrm{CD} 71^{\text {low }} / \mathrm{CD} 24 \mathrm{a}^{\text {low }}$ cells using the additional markers CD150, CD9 and Sca1, where sBFU-E mediated recovery from irradiation-induced acute anemia by providing a transient wave of erythroid cells in the $\mathrm{PB}$ and spleen, followed by multi-lineage reconstitution in the PB, spleen and BM from sMPP.

\section{Stress-BFU-E and stress-CFU-E display opposing expres- sion patterns of BMP- and CBFA2T3-responsive genes}

To identify gene expression patterns associated with the distinct features of the radio-protective sBFU-E, RNAsequencing was performed on FACS sorted stress-progenitor populations from day 8 spleens. Unsupervised clustering of genes with significant differential expression between any of the investigated populations revealed that relatively few genes were uniquely expressed by sBFU-E, about half of the genes differing between progenitor populations were shared between sMPP and sBFU-E (Figure $3 \mathrm{~A}$, gene lists for each cluster in the Online Supplemental Table S1), while the sCFU-E were clearly distinct from the other two populations. Gene set enrichment analysis $(G S E A)^{24}$ revealed that genes that were uniquely up-regulated in sMPP were associated with adult tissue stem cells and long-term hematopoietic stem cells (Figure 3B), while genes up-regulated in sBFU-E were associated with MAPK signaling, response to stress and stimulus, and more mature hematopoietic progenitors (Figure $3 \mathrm{~B}$ ). Interestingly, both sMPP and sBFU-E expressed BMP- as well as CBFA2T3 target genes known to be up-regulated in response to Bmp or Cbfa2t3 inactivation respectively (Figure $3 \mathrm{~B}$ ). sCFU-E on the other hand, uniquely expressed genes correlating with late progenitors, heme metabolism, and BMP- and CBFA2T3-responsive genes reported to be down-regulated in response to $\mathrm{Bmp}$ or $\mathrm{Cbfa} 2 \mathrm{t} 3$ inactivation (Figure $\mathrm{BB}$ ).

The differential expression of BMP-responsive genes in sCFU-E and more primitive stress-progenitors is well in line with previous findings showing that effective generation of erythroid stress-progenitors is BMP-dependent. To explore
Table 2. Top 20 down-regulated genes in stress-BFU-E compared to steady-state.

\begin{tabular}{|c|c|c|}
\hline Gene symbol & log2 fold change & $-\log 10$ FDR \\
\hline Fl3al & $-2,754$ & 29,923 \\
\hline Snord15a & $-2,519$ & 23,305 \\
\hline Tifab & $-2,413$ & 16,746 \\
\hline Csflr & $-2,358$ & 16,322 \\
\hline$C y b b$ & $-2,344$ & 13,756 \\
\hline Unc93b1 & $-2,321$ & 25,436 \\
\hline Lgals1 & $-2,265$ & 14,959 \\
\hline Cf10 & $-2,250$ & 14,576 \\
\hline Snord15b & $-2,197$ & 43,032 \\
\hline Rasa4 & $-2,127$ & 13,580 \\
\hline$C d 209 a$ & $-2,124$ & 10,439 \\
\hline Klf4 & $-2,115$ & 10,450 \\
\hline Lrpl & $-2,024$ & 9,143 \\
\hline Pik3r5 & $-1,939$ & 8,927 \\
\hline Itsn1 & $-1,907$ & 21,849 \\
\hline $\mathrm{Ccr} 2$ & $-1,860$ & 7,775 \\
\hline$L y 6 c 2$ & $-1,859$ & 7,727 \\
\hline Trim47 & $-1,847$ & 8,101 \\
\hline Nhsl2 & $-1,796$ & 7,029 \\
\hline Irf8 & $-1,783$ & 7,515 \\
\hline
\end{tabular}

The genes most down-regulated in sBFU-E compared to steady-state included the coagulation factor F13a1/FxIIIa expressed by monocytes and megakaryocytes, Tifab a del(5q) MDS gene known to regulate hematopoiesis and mediate immune signaling through the Toll-like receptor-TRAF6 pathways, ${ }^{41}$ the Kit paralog Csflr; essential for the survival of monocytes and macrophages, ${ }^{42}$ and Cybb/gp91-phox; a heme-binding membrane glycoprotein that is a phagocyte respiratory burst oxidase component induced by inflammation. ${ }^{43}$

the functional importance of BMP-signaling for the generation of stress-progenitor populations, BM cells deficient in $\mathrm{BMP}$ receptor II from conditional knock out mice (BmprIIfl/fl Vav-Cre) or wild-type littermate controls (BmprIIfl/fl Cre-negative) were competitively transplanted at a 1:1 ratio with $\mathrm{KuO}^{+}$wild-type $\mathrm{BM}$, and analyzed for stress recovery contribution in the spleen on day 8 (Figure 3C). Mice transplanted with BMPRII deficient BM had smaller spleens and a 36\% reduction in cells/spleen (Figure 3D), despite the fact that $50 \%$ of the transplanted BM cells were wild-type. This difference was no longer apparent after lymphoprep-enrichment of mononuclear cells, indicating that spleens of mice transplanted with 100\% wild-type cells contained more erythroid cells (Figure 3D). Accordingly, BMPRII-deficient BM displayed a substantially decreased potential to form stress-progenitors from the stage of lineage negative $\mathrm{cKit}^{+}$progenitors $(59.6 \pm 5,0 \%$ of wild-type), with the most prominent reduction observed in the number of sBFU-E $(46.4 \pm 6.7 \%$ of wild-type) (Figure $3 \mathrm{E}$, see Figure 1C for gating). Hence, in agreement with previous studies, our prospectively isolated stress-progenitor populations are dependent on BMP signaling for a full stress-response.
CD150 and CD9 mark BFU-E potential during steady-state erythropoiesis, providing improved identification of myelo-erythroid progenitors in the BM
Mapping transcriptional profiles of the splenic stress- progenitor populations (see Figure 3 ) to transcriptional pro- 
files of steady-state progenitors from BM as recently defined by single-cell transcriptomics and fate assays, ${ }^{15}$ demonstrated that splenic stress-progenitors map closely with their steady-state BM counterparts (Online Supplementary Figure S6). The most widely used protocol for delineating myelo-erythroid progenitors in steady-state BM nicely demonstrates that BFU-E potential resides in the $\mathrm{Lin}^{-} \mathrm{CKit}^{+} \mathrm{CD} 150^{+} \mathrm{CD} 105^{+}$"Pre-CFU-E" fraction, ${ }^{9}$ also confirmed in our hands (Figure 4A-B). However, since the "PreCFU-E" population contains relatively few BFU-E progenitors (Figure 4B and Pronk et al.), ${ }^{9}$ a specific cell population possessing the BFU-E potential remains poorly defined. We therefore asked if CD9 could be used to also enrich for steady-state BFU-E. Progenitor populations were FACS-sorted from steady-state BM using the same markercombinations as in stressed spleen $\left(\mathrm{CD} 150^{+} \mathrm{CD} 9^{+} \mathrm{Sca} 1^{-}\right)$, and plated for erythroid colony formation. The FACS profile of steady-state BM was very similar to that of stressed spleen (Figure 1C and Figure 4C), and both Sca1 and Sca1 ${ }^{+}$ $\mathrm{CD} 150^{+} \mathrm{CD}^{+}{ }^{+} \mathrm{BM}$ cells efficiently gave rise to BFU-E colonies (BFU-E/CFU-E colony formed from CD150+CD9 ${ }^{+}$Sca1 ${ }^{-}: 30.0$, frequency: $7.5 \pm 0.9 \%$, Figure 4CD), representing a 45 -fold improved BFU-E/CFU-E ratio compared to previously used marker combination. In contrast, $\mathrm{CD} 150^{+} \mathrm{CD}^{+}$cells were hardly present in spleens from steady-state mice, and of these only a few comprised BFU-E-forming potential (BFU-E/CFU-E colony formed

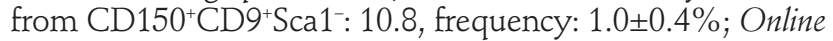
Supplementary Figure S7). Furthermore, steady-state BM progenitors gave rise to highly proliferative BFU-E colonies, whereas steady-state spleen progenitors resulted in much smaller BFU-E colonies (data not shown). In conclusion, CD150 and CD9 expression mark BFU-E potential, both during steady-state in the BM and in the spleen during acute anemia.

\section{Compared to steady-state BFU-E, stress-BFU-E have enhanced expression of genes associated with BMP sig- naling, erythropoiesis and proliferation}

To investigate prospective mechanisms giving stressBFU-E their unique capacity to rapidly produce large numbers of erythroid cells in response to anemia, BFU-E $\left(\mathrm{CD}_{150}{ }^{+} \mathrm{CD}^{+} \mathrm{Sca}^{-}\right)$were sorted from steady-state BM and day 8 stressed spleens and analysed for transcriptional differences using RNA-seq. Analysis demonstrated a large overlap between stress- and steady-state BFU-E, with only 277 genes being differentially expressed (Figure 5A, full list of differentially expressed genes in the Online Supplemental Table S2). Gene set enrichment analysis demonstrated that sBFU-E expressed higher levels of genes associated with $\mathrm{BMP}$ and glucocorticoid signaling, proliferation, maturation block, and erythropoiesis (Figure $5 \mathrm{~B}$ and Online Supplementary Figure S8) with several erythropoiesis-related genes among the most highly up-regulated genes in stress- compared to steady-state BFU-E (Table 1). Steadystate BFU-E on the other hand retained gene signatures associated with myeloid cell development and immune response (Figure 5C), which was also reflected by the genes most down-regulated in stress- compared to steady-state BFU-E (Table 2).

Strong prevalence for the binding motif of chromatin-looping transcription factor CTCF under ATACseq peaks enriched in stress-BFU-E

To define potential differences in active regulatory DNA elements across the genome, we performed the assay for transposase-accessible chromatin sequencing (ATAC-seq) analysis ${ }^{18}$ on the same stress- and steady-state BFU-E populations as used for transcriptional profiling. ATAC-seq peaks (open chromatin regions) were detected using ENCODE ATAC-seq pipeline (https://github.com/kundajelablatac_dnase_pipelines). K-means clustering demonstrated a relatively large overlap in chromatin availability between the two populations, with some of the peaks being enriched in stress- (orange) or steady-state (blue) BFU-E (Figure 5D and Online Supplementary Figure S9). The absolute majority of peaks with differential accessibility were located at distal elements $(>1,000 \mathrm{bp}$ away from the transcription start site) with only $5 \%$ situated around the promoter regions in both stress- and steady-state BFU-E, indicating that transcriptional differences between stressand steady-state BFU-E are likely to be regulated by distal chromatin interactions.

To identify DNA-binding factors with differential chromatin availability in stress-erythropoiesis we performed motif analysis using peak files extracted from the heatmap clusters. This detected the transcriptional regulator CTCF (CCCTC-binding factor, CTCFL; testis-specific paralog) as the most significantly enriched DNA-binding factor in stress-BFU-E peaks followed by ERG (Figure 5E), whereas steady-state BFU-E peaks most significantly enriched for SFBPI1 and RUNX2 (Figure 5F). Notably, only for CTCF was the motif distributed closely around the peak center (Figure 5G). CTCF is known to regulate the formation of chromatin loops by binding together strands of DNA, and also constitutes a primary part of insulators by blocking the interaction between enhancers and promoters (reviewed by Phillips et al.). ${ }^{29}$ Interestingly, CTCF has been shown to mark active promoters and boundaries of repressive chromatin domains in primary human erythroid cells, ${ }^{30}$ and LDB1-CTCF enhancer looping has recently been shown to underlie activation of a substantial fraction of erythroid genes. $^{31}$ Furthermore, CTCF has been shown to regulate growth and erythroid differentiation of human myeloid leukemia cells (K562 cell line). ${ }^{32}$ Although both steady-state and stress-BFU-E enriched for the CTCF motif, the frequency was considerably higher in sBFU-E, where $24,3 \%$ of the enriched peaks marked a CTCF motif, compared to $8,5 \%$ in steady-state BFU-E (Figure $5 \mathrm{H}$ ). In conclusion, while the transcriptional and chromatin landscape of stress- and steady-state BFU-E display a high degree of similarity, distinctions in gene expression patterns and in the epigenetic landscape might underlie the unique capacity of sBFU-E to rapidly make large numbers of erythroid cells in response to anemia.

\section{Discussion}

The identity of stress-erythroid progenitors has remained largely elusive, and their precise identification is important to understand the mechanisms governing recovery from anemia. Previous studies have identified stress-progenitors to be $\mathrm{cKit}{ }^{+} \mathrm{CD} 71 \mathrm{low}^{\mathrm{ow}} / \mathrm{Ter} 119^{\mathrm{ow}},{ }^{14}$ and after culture the BFU-E potential is found in the $\mathrm{CD} 34^{-} \mathrm{CD} 133$ fraction of the $\mathrm{Sca} 1^{+} \mathrm{CKit}{ }^{+} \mathrm{CD} 71^{\text {low }} / \mathrm{Ter} 119^{\text {low }}$ population. However, only a small fraction of these cells $(0.12-0.2 \%)$ gave rise to BFU-E colonies. Here we demonstrate that the differential expression of the surface markers CD150, CD9 and Sca1 provide fractionation and definition of a 
hierarchy of functionally diverse splenic stress-progenitors during irradiation-induced recovery, providing 100-fold improved enrichment of stress-BFU-E compared to the current state-of-the-art.

Analysis of megakaryocytic-erythroid differentiation kinetics has been hampered by the lack of markers expressed by mature erythrocytes and platelets. By transplanting sorted stress-progenitor populations from transgenic mice expressing Kusabira Orange in all cells including erythrocytes and platelets, ${ }^{20}$ we determined their kinetics and full differentiation potential in vivo. Although sBFU-E gave rise to megakaryocytic colonies in vitro and $\mathrm{CD} 41^{+}$cells in vivo, only sMPP produced platelets in vivo, demonstrating the importance of in vivo experiments and the ability to trace all mature cell types for defining progenitor potential. In agreement with previous studies, ${ }^{33}$ this shows that CD41 is not specific for the megakaryocytic lineage.

We further demonstrate that splenic sBFU-E provide a massive but transient erythroid wave, followed by multilineage reconstitution from sMPP. Harandi et al. ${ }^{14}$ previously suggested that $\mathrm{cKit}^{+} \mathrm{CD} 71^{-}$Ter119- cells were erythroid restricted..$^{14}$ However, using the same starting population of cells and in vivo reconstitution we now demonstrate that these cells contain both multi-potent progenitors $\left(\mathrm{CD} 150^{+} \mathrm{CD} 9^{+} \mathrm{Sca}^{+}\right)$and more erythroid restricted BFU-E $\left(\mathrm{CD} 150^{+} \mathrm{CD} 9^{+} \mathrm{Sca1}^{-}\right)$and CFU-E (CD150 $\left.{ }^{+} \mathrm{CD} 9^{-}\right)$. The same group also proposes that stress-BFU-E are Sca1 ${ }^{+}$, whereas we show that Sca1 ${ }^{+}$stress-progenitors are multi-potent with the capacity to give rise to more restricted Sca- sBFUE. Sca1 is known to mark multi-potent HSC, and that Sca1 as a single surface marker can differentiate between multilineage and BFU-E potential is well in line with cellular hierarchy mapping in steady-state hematopoiesis. ${ }^{34}$ Interestingly, CD9 has recently been reported to be expressed in murine HSC with MegE differentiation bias. ${ }^{34}$ While Pronk et al..$^{9}$ have identified that CD150 marks "PreCFU-E" colony-forming potential in the BM during steady-state, we for the first time describe a FACS method for identifying progenitors with BFU-E potential, which resides in the $\mathrm{cKit}{ }^{+} \mathrm{CD} 71^{\text {low }} / \mathrm{CD} 24 \mathrm{a}^{\text {low }} \mathrm{CD} 150^{+} \mathrm{CD} 9^{+}$population. This demonstrates that these markers can be used to enrich for both steady-state BFU-E and stress-BFU-E.

BMP-regulation of stress-erythropoiesis has previously been described by Paulson and colleagues, whereas we have shown that steady-state erythropoiesis remains unaffected by disruption of canonical BMP-signaling. Investigation of the functional importance of BMP-signaling in our system revealed that transplantation of BMP-deficient BM resulted in an impaired stress-response with substantially smaller spleens and decreased potential to form stress-progenitors, despite $50 \%$ of the transplanted BM cells being wild-type. In addition, genes upregulated in sBFU-E compared to their steady-state counterpart were associated with gene sets activated downstream of the BMP signaling pathway. ${ }^{33}$ Within the stress-erythroid progenitor populations, sCFU-E expressed the same set of BMP-responsive genes to a higher degree than sBFU-E and sMPP. Taken together, these results model stress-erythroid progenitors in general and sCFU-E in particular as BMP-responsive cells. SCFU-E also displayed the highest expression of a set of genes that is down-regulated in response to inactivation of Cbfa2t $3{ }^{26}$ a transcriptional co-repressor that promotes degradation of hypoxia regulating protein Hifla, ${ }^{35}$ regulates Gata1-target genes critical for erythroid differentiation, ${ }^{36}$ and maintains an erythroid-specific genetic program in progenitors primed for rapid activation when terminal erythroid differentiation is induced. ${ }^{37}$ Stress-erythropoiesis is severely impaired in mice lacking Cbfa $2 \mathrm{t} 3 .^{26}$ Collectively, this makes Cbfa2t 3 an interesting target to study further in stress-erythropoiesis regulation.

Analysis of active regulatory DNA elements across the genome using ATAC-seq revealed that sBFU-E displayed a stronger prevalence for the binding motif of chromatin-looping transcription factor CTCF compared to steady-state BFU-E which are less efficient in producing erythrocytes. CTCF has previously been implicated in marking active promoters in primary human erythroid cells ${ }^{30}$ and LDB1-CTCF enhancer looping underlies activation of a substantial fraction of erythroid genes. ${ }^{31}$ In accordance, ectopic expression of CTCF in K562 cells promotes erythroid differentiation whereas CTCF knock-down significantly inhibits differentiation into the erythroid lineage. ${ }^{32}$ Taken together, chromatin accessibility of CTCF is the most striking epigenetic difference between stress- and steady-state BFU-E, suggesting a role for CTCF-dependent mechanisms in stress-BFU-Es.

In conclusion, combining novel surface markers with the $\mathrm{KuO}$ tracing mouse, we have for the first time defined a cellular hierarchy of stress-progenitors, separating sMPP from sBFU-E and more mature sCFU-E based on their kinetics and differentiation potential in vivo. We further demonstrate that sBFU-E express gene signatures more associated with erythropoiesis and proliferation compared to steady-state BFU-E, and have enhanced and differential accessibility to CTCF binding sites. Our findings open up the field for further mechanistic and functional studies of how stress-erythropoiesis is regulated, and how sBFU-E contribute during recovery of erythroid disorders. Since the mechanisms regulating stress-erythropoiesis may be targeted for treatment of anemia, this is an important step towards identifying and studying novel erythropoiesis stimulating agents.

\section{Acknowledgments}

The authors would like to thank Dr. Hiromitsu Nakauchi for kindly providing the Kusabira Orange mice.

\section{Funding}

This research was supported by the Ragnar Söderberg Foundation (fellowship JF), the Swedish Cancer Society (fellowship SS), the Swedish Research Council and the Swedish Foundation for Strategic Research (JF), the Swedish Foundation for Medical Research, the Crafoordska Foundation, the Ake Wiberg Foundation, the Clas Groschinsky's Memory Foundation and the Harald \& Greta Jeansson Foundation (SS).

\section{References}

1. Lenox LE, Perry JM, Paulson RF. BMP4 and Madh5 regulate the erythroid response to acute anemia. Blood. 2005;105(7):27412748.
2. Peslak SA, Wenger J, Bemis JC, et al. EPOmediated expansion of late-stage erythroid progenitors in the bone marrow initiates recovery from sublethal radiation stress. Blood. 2012;120(12):2501-2511.

3. Harandi OF, Hedge S, Wu DC, McKeone D, Paulson RF. Murine erythroid short-term radioprotection requires a $\mathrm{BMP} 4$-dependent, self-renewing population of stress erythroid progenitors. J Clin Invest. 2010 120(12):4507-4519.

4. Perry JM, Harandi OF, Paulson RF. BMP4, SCF, and hypoxia cooperatively regulate the expansion of murine stress erythroid pro- 
Isolation and molecular signature of stress-BFU-Es

5. Singbrant S, Karlsson G, Ehinger M, et al. Canonical BMP signaling is dispensable for hematopoietic stem cell function in both adult and fetal liver hematopoiesis, but essential to preserve colon architecture. Blood. 2010;115(23):4689-4698.

6. Singbrant S, Moody JL, Blank U, et al. Smad5 is dispensable for adult murine hematopoiesis. Blood. 2006;108(12):37073712.

7. Paulson RF, Shit L, Wu DC. Stress erythropoiesis: new signals and new stress progenitor cells. Cor Spin Hematol. 2011; 18(3):139-145.

8. Chang J, Socolovsky M, Gross AW, Lodish HF. Role of Res signaling in erythroid differentiation of mouse fetal liver cells: functional analysis by a flow cytometry-based novel culture system. Blood. 2003;102(12):39383946.

9. Pronk CJ, Ross DJ, Manson R, et al. Elucidation of the phenotypic, functional, and molecular topography of a myeloerytheroid progenitor cell hierarchy. Cell Stem Cell. 2007;1(4):428-442.

10. Bu J, Lu J, Que F, et al. Isolation and functional characterization of human erythroblasts at distinct stages: implications for understanding of normal and disordered erythropoiesis in vino. Blood. 2013; 121(16):3246-3253.

11. Li J, Hale J, Bhagia P, et al. Isolation and transcriptome analyses of human erythroid progenitors: BFU-E and CFU-E. Blood. 2014; 124(24):3636-3645.

12. Kingsley PD, Greenfest-Allen E, Frame JM, et al. Ontogeny of erythroid gene expresssion. Blood. 2013;121(6):e5-e13.

13. Pishesha N, Thru P, Shy J, Eng JC, Sankaran VG, Lodish HF. Transcriptional divergence and conservation of human and mouse errthropoiesis. Proc Natl Acad Sci U S A. 2014; 111(11):4103-4108.

14. An X, Schulz VP, Li J, et al. Global transcriptome analyses of human and murine terninat erythroid differentiation. Blood. 2014; 123(22):3466-3477.

15. Tui BK, Wolock SL, Weinreb C, et al. Population snapshots predict early haematopoietic and erythroid hierarchies. Nature. 2018;555(7694):54-60.

16. Flygare J, Rayon Estrada V, Shin C, Gupta S, Lodish HF. HIF1alpha synergizes with glucocorticoids to promote BFU-E progenitor selfrenewal. Blood. 2011;117(12):3435-3444.

17. Kiang J, Wu DC, Chen Y, Paulson RF. In vito culture of stress erythroid progenitors idemtifies distinct progenitor populations and analogous human progenitors. Blood. 2015;125(11):1803-1812.

18. Buenrostro JD, Giresi PG, Zaba LC, Chang
HY, Greenleaf WJ. Transposition of native chromatin for fast and sensitive epigenomic profiling of open chromatin, DNA-binding proteins and nucleosome position. Nat Methods. 2013;10(12):1213-1218.

19. Porayette P, Paulson RF. BMP4/Smad5 dependent stress erythropoiesis is required for the expansion of erythroid progenitors during fetal development. Lev Biol. 2008; 317(1):24-35.

20. Hamanaka S, Ooehara J, Morita Y, et al. Generation of transgenic mouse line expressing Kusabira Orange throughout body, including erythrocytes, by random segregation of provirus method. Biochem Biophys Res Commune. 2013;435(4):586591.

21. Till J, McCulloch E. A direct measurement of the radiation sensitivity of normal mouse bone marrow cells. Radial Res. 1961; 14(2):213-222

22. Na Nakorn $\mathrm{T}$, Trave $\mathrm{D}$, Weissman IL, Akashi K. Myeloerythroid-restricted progenitors are sufficient to confer radioprotectron and provide the majority of day $8 \mathrm{CFU}$ S. J Chin Invest. 2002;109(12):1579-1585

23. Siminovitch L, McCulloch EA, Till JE. The distribution of colony-Forming cells among spleen colonies. J Cell Physiol. 1963; 62(3):327-336.

24. Subramanian A, Tamayo P, Mootha VK, et al. Gene set enrichment analysis: a knowledge-based approach for interpreting genome-wide expression profiles. Proc Natl Acad Sci U S A. 2005;102(43):15545-15550.

25. Lee KY, Jeong JW, Wang J, et al. Bmp2 is critital for the murine uterine decidua response. Mol Cell Biol. 2007;27(15):54685478.

26. Chyla BJ, Moreno-Miralles I, Steapleton $\mathrm{MA}$, et al. Deletion of Mtg16, a target of $t(16 ; 21)$, alters hematopoietic progenitor cell proliferation and lineage allocation. Mol Cell Biol. 2008;28(20):6234-6247.

27. Lenox LE, Shy L, Hegde S, Paulson RF. Extramedullary erythropoiesis in the adult liver requires BMP-4/Smad5-dependent sighaling. Exp Hematol. 2009;37(5):549-558.

28. Perry JM, Harandi OF, Porayette P, Hegde S, Kennan AK, Paulson RF. Maintenance of the BMP4-dependent stress erythropoiesis pathway in the murine spleen requires hedgehog signaling. Blood. 2009;113(4):911-918.

29. Phillips JE, Cores VG. CTCF: master weaner of the genome. Cell. 2009;137(7):11941211.

30. Steiner LA, Schulz V, Makismova Y, LezonGeyda K, Gallagher PG. CTCF and CohesinSA-1 mark active promoters and boundaries of repressive chromatin domains in primary human erythroid cells. PLoS One. 2016;11(5):e0155378.
31. Lee J, Krivega I, Dale RK, Dean A. The LDB1 Complex co-opts CTCF for erythroid lineage-specific long-range enhancer interacttons. Cell Rep. 2017;19(12):2490-2502.

32. Torrano V, Chernukhin I, Docquier F, et al. CTCF regulates growth and erythroid differentiation of human myeloid leukemia cells. J Biol Chem. 2005;280(30):28152-28161.

33. Gekas C, Graf T. CD 41 expression marks myeloid-biased adult hematopoietic stem cells and increases with age. Blood. 2013; 121(22):4463-4472.

34. Gro G, Luc S, Marco E, et al. Mapping celllar hierarchy by single-cell analysis of the cell surface repertoire. Cell Stem Cell. 2013; 13(4):492-505.

35. Kumar P, Gullberg U, Olson I, Ajore R. Myeloid translocation gene-16 co-repressor promotes degradation of hypoxia-inducible factor 1. PLo One. 2015;10(5):e0123725.

36. Fujiwara T, Alqadi YW, Okitsu Y, et al. Role of transcriptional corepressor ETO2 in errthroid cells. Exp Hematol. 2013;41(3):303315.

37. Stadhouders R, Cisco A, Stephen T, et al. Control of developmentally primed rythroid genes by combinatorial co-repressor actions. Nat Commune. 2015;6:8893.

38. Baker WJ, van Dijk TB, Parren-van Amelsvoort M, et al. Differential regulation of Foxo3a target genes in erythropoiesis. Mol Cell Biol. 2007;27(10):3839-3854.

39. Roy A, Lordlier L, Mazzi S, et al. Activity of nonmuscle myosin II isoforms determines localization at the cleavage furrow of megakaryocytes. Blood. 2016;128(26):31373145 .

40. Mirabelli P, Di Nato R, Lo Pardo C, et al. Extended flow cytometry characterization of normal bone marrow progenitor cells by simultaneous detection of aldehyde denydrogenase and early hematopoietic antigens: implication for erythroid differentiation studies. BMC Physiol. 2008;8:13.

41. Varney ME, Niederkorn M, Konno H, et al. Loss of Tifab, a del (sq) MDS gene, alters hematopoiesis through derepression of Tolllike receptor-TRAF6 signaling. J Exp Med. 2015;212(11):1967-1985.

42. Chitu V, Stanley ER. Colony-stimulating factor -1 in immunity and inflammation. Cor Open Immunol. 2006;18(1):39-48.

43. Belambri SA, Roles L, Rad H, HurtadoNedelec M, Dang PM, El-Benna J. NADPH oxidase activation in neutrophils: role of the phosphorylation of its subunits. Eur J Chin Invest. 2018;48 Suppl 2:e12951.

44. Heinz S, Benner C, San N, et al. Simple combinations of lineage-determining transcription factors prime cis-regulatory lemints required for macrophage and $\mathrm{B}$ cell identities. Mol Cell. 2010;38(4):576-589.

haematological | 2020; 105(11)

2571 